\title{
Evaluación de la satisfacción de alumnos de educación superior del Centro Universitario Sur de la Universidad Autónoma de Tamaulipas (Evaluation of the satisfaction of students of higher education of the South University Center of the Universidad Autonoma de Tamaulipas)
}

\section{Giovanna Castañeda Hernández Gustavo Alarcón Martínez*}

\begin{abstract}
The purpose of the research is to define the influence of clue factors in the satisfaction perceived by students of Centro Universitario Sur de la Universidad Autónoma de Tamaulipas. Interview of 90 students allows determining the grade of influence of those clue factors. This research includes a statistical analysis as well as regression analysis. In order of importance results indicates that supporting services, infrastructure, teacher performance and teach- and- learn competences, have a positive relation in respect to satisfaction perceived by students. These results could be useful for higher education institutions planning.
\end{abstract}

Key words: higher education, quality service, regression, student's perception

JEL: I23, L80.

Resumen. El propósito de esta investigación es determinar la influencia de factores claves en la satisfacción percibida por los alumnos del Centro Universitario Sur de la Universidad Autónoma de Tamaulipas. Una encuesta a una prueba piloto de 90 alumnos permitió determinar en qué grado inciden los factores clave. La investigación incluye un análisis estadístico y de regresión, los resultados indican que los estudiantes consideran que, en orden de importancia, los servicios de apoyo, la infraestructura, el desempeño del docente y las unidades de enseñanza aprendizaje representan una relación positiva con su satisfacción. Estos resultados pueden ser de utilidad para la planeación que realizan las instituciones de educación superiorlas variables y una disparidad de la percepción entre las empresas extranjeras y mexicanas.

Palabras clave: educación superior, percepción, regresión lineal múltiple, servicio de calidad

\footnotetext{
- Universidad Autónoma de Tamaulipas, Facultad de Derecho y Ciencias Sociales, Campus Tampico, México. Email: giovanna_castaneda@hotmail.com

* Universidad Autónoma de Nuevo León. Facultad de Contaduría Pública y Administración, San Nicolás de los Garza, N.L., México. Email: gusalamar@hotmail.com
} 


\section{Introducción}

Uno de los criterios para evaluar la calidad de los servicios que brindan las IES, es la valoración que los usuarios otorgan a los servicios que reciben de dicha institución (Alvarezet al., 2015). Algunos estudios centran su interés en conocer las expectativas que tienen los estudiantes universitarios acerca de las condiciones para mejorar su proceso educativo. Las investigaciones realizadas en este tema, se clasifican en dos tipos: las que se realizan para saber las expectativas del estudiante con respecto al servicio que ofrece la universidad en general y aquellas que estudian las expectativas de los estudiantes sobre el proceso de enseñanza- aprendizaje (Pichardo et al., 2007). Por lo que respecta a esta investigación se consideran las expectativas del estudiante sobre el servicio que le proporciona la universidad.

De acuerdo con Mejías \& Martínez (2009), medir la satisfacción del cliente tiene sentido siempre que se acompañe de acciones que induzcan la mejora y la innovación; es por ello que medir la satisfacción de los estudiantes de una manera consistente, permanente y adecuada, orientaría a la toma de decisiones que permitan incrementar las fortalezas y subsanar las debilidades de las Instituciones de Educación Superior.

\section{Planteamiento del problema de investigación}

El estudio de la satisfacción de los alumnos como clientes del servicio educativo que les brinda el sistema de educación superior es un reto que ha sido abordado desde diferentes perspectivas. En el caso particular del estado de Tamaulipas el Gobierno del Estado (2017) ha señalado que tiene que ver con promocionar y difundir el acceso a una educación de calidad, mediante incentivos y acciones formativas que contribuyan a disminuir el rezago educativo, aumentar la eficiencia terminal y mejorar el aprovechamiento escolar, así como a aplicar medios, métodos y mecanismos de seguimiento y evaluación a la calidad educativa.

Sin embargo, a pesar de que este tema es recurrente en los distintos espacios de la sociedad tamaulipeca por la importancia que reviste, no se han encontrado estudios que se refieran a las instituciones de educación superior que aborden desde una misma metodología esta problemática, por 
lo cual nace esta propuesta de investigación con la inquietud de proponer variables que incidan en la satisfacción de los alumnos de las instituciones de educación superior.

Así, el objetivo de esta investigación es realizar una revisión de la literatura en el contexto global que sustente la existencia de factores que inciden en la satisfacción del cliente, en este caso alumnos de las Instituciones de Educación Superior, y posteriormente llevar a cabo un análisis cuantitativo de la importancia de dichos factores, en el caso particular del Centro Universitario de la Zona Sur de Tamaulipas.

\section{Antecedentes teóricos del fenómeno a estudiar}

Estudios sobre la calidad educativa se han enfocado principalmente en identificar los factores que inciden en la calidad. Por mencionar algunos Weinstein (2002) señala que existen factores críticos de una gestión escolar de calidad que permiten mejores resultados pedagógicos, entre los que menciona; liderazgo, trabajo en equipo, nivel de compromiso con los profesores, proceso de planificación institucional, participación efectiva de los distintos actores del sistema educativo, clima laboral positivo entre docentes, directivos y alumnos, y una adecuada inserción.

Brunner \& Elacqua (2004) identifican un conjunto de factores que inciden en una educación de calidad, entre ellos: liderazgo y cooperación; clima de aprendizaje; monitoreo continuo del progreso de los alumnos; evaluación frecuente del desempeño de sus profesores; profesores reconocidos por su desempeño y gestión autónoma.

Por su parte Faganel \& Dolinsek (2012) mencionan que la calidad en la enseñanza de educación superior se deben incorporar elementos como evaluaciones internas sobre la institución y sus programas donde participen los estudiantes, con la finalidad de satisfacer los requerimientos de los empleadores. Por otra parte, González et al. (2017) mencionan que la evaluación de los planes de calidad universitarios se optimiza cuando éstos incorporan opiniones e indicadores de satisfacción estudiantil. Esta investigación se refiere a diversos factores relacionado con la satisfacción de los estudiantes. 


\section{El fenómeno a estudiar: La satisfacción del alumno}

Algunos estudios en educación superior han trasladado y utilizado los modelos de Parasuraman et al. (1985), con la finalidad de crear instrumentos que permitan establecer conclusiones en el estudio de la calidad del servicio en universidades. Por su parte Pichardo et al. (2007) consideran que esta perspectiva promueve estudios encaminados a conocer y determinar las expectativas de los estudiantes universitarios, como punto de partida para mejorar la calidad del servicio ofrecido por las instituciones de educación superior entre los que destacan Hill (1995); Narasimhan (1997); Sander et al. (2000) Keogh \& Stevenson (2001) Darlaston-Jones et al. Drew (2003).

En la actualidad las universidades se interesan por encontrar nuevas formas para mejorar los servicios educativos que ofrecen a la comunidad tras observar si estos son de calidad, y en efecto satisface las partes involucradas en el proceso, lo cual ha llevado a revisar estudios cuyo objetivo es medir la satisfacción del estudiante. Entre estos destaca el de Jiménez et al. (2011) que en su investigación aplican un cuestionario con la finalidad de conocer el grado de satisfacción de los estudiantes respecto al plan de estudios que están cursando, donde consideran aspectos como: a) unidad de aprendizaje, b) metodología, c) infraestructura, d) desempeño de los profesores y e) desempeño de los estudiantes.

En el ámbito internacional, se han realizado algunas investigaciones cuyo objeto de estudio es conocer la percepción de la satisfacción de los estudiantes de nivel superior. En Venezuela, se realizó un estudio aplicando un instrumento que considera los siguientes aspectos: a) servicios, b) necesidades básicas, c) seguridad, d) seguridad económica, e) seguridad emocional, f) pertenencia a la institución o grupo de alumnos, g) sistema de trabajo, h) progreso del éxito personal, i) reconocimiento del éxito y j) autorrealización personal (Gento \& Vivas, 2003).

Por otra parte Alvarez et al. (2015) realizan un estudio que les permite conocer la satisfacción del estudiante en relacion a los servicios educativos que les proporciona la Universidad Valle de Toluca, para dicho estudio aplicaron una encuesta en escala de likert donde consideraron aspectos como: a) plan de estudios, b) capacitación y habilidad para la enseñanza de los docentes, c) métodos de enseñanza y evaluación, d) nivel de 
autorrealización de los estudiantes, e) servicios de apoyo, f) servicios administrativos, g) ambiente propicio e infraestructura.

En relación a la percepción que tiene los estudiantes sobre la calidad de los servicios que reciben de una institucion privada, en comparación con una de caracter público, Alvarado et al. (2016) elaboraron un estudio considerando los siguientes factores: a) la infraestructura física, b) el proceso enseñanza - aprendizaje que lleva a cabo la planta docente, c) la habilidad para transmitir el conocimiento, y d) el desarrollo integral que fomentan en los estudiantes los programas académicos.

Para efectos de la presente investigación, la satisfaccion del estudiante se entendera como la percepción que tiene los estudiantes de educación superior sobre el total de los servicios y apoyos que le proporciona la universidad.

\section{Los factores que influyen en el fenómeno}

Los factores que influyen en la satisfacción de los estudiantes en el caso de esta investigación tiene como base las consideraciones de Jiménez, Terriquez \& Robles (2011), Gento \& Vivas (2003), Alvarez et al. (2015), Alvarado et al. (2016) y son los siguientes: unidades de enseñanza aprendizaje, metodología de evaluación del proceso de enseñanaza aprendizaje, desempeño de los profesores, servicio de apoyo e infraestructura.

\section{Unidades de enseñanza - aprendizaje}

Bajo este contexto se han consultado diversos autores que han trabajado con estos conceptos entre los cuales podemos citar a Díaz (2014) quien menciona que un programa de estudios debe contener una breve descripción de la asignatura, módulo o unidad de aprendizaje, donde se incluye la organización en unidades o bloques, así como la evaluación y la bibliografía. Por otra parte Zabalza (1987) consideran que el curricula debe contener los contenidos de aprendizaje que se pretende que los estudiantes vayan adquiriendo, su organización, es decir qué contenidos se abordarán en cada bloque, así como la metodología y la evaluación que permita constatar si el proceso en curso cumplió las expectativas. 
En relación a lo anterior, para el desarrollo de esta investigación se consideran unidades de eneñanza - aprendizaje, a aquellos progamas de estudio, progamas de asignatura o curricula que se detallan en el documento que desglosa los contenidos de la materia por bloques y la metodología de evaluación de los contenidos.

\section{Metodología de evaluación del proceso enseñanza - aprendizaje.}

En relación a la metodología de evaluación del proceso enseñanza aprendizaje, este factor permite constar que el proceso de aprendizaje ha cumplido sus expectativas (Zabalza,1987). Hamodi et al. (2015) definen como medios de evaluación las producciones del alumno que permiten evidenciar lo que han aprendido. Santos (1993) señala que evaluar es dar valor a las cosas. En concordancia con lo anterior, la metodología de evaluación se define como el valor que se le asigna a las producciones del alumno con la finalidad de constatar lo aprendido.

\section{Desempeño del docente}

En el ámbito educativo se realiza la evaluación del desempeño de los profesores, mediante el desarrollo de las competencias profesionales del docente de educación superior, las cuales se pueden definir como el conjunto de conocimientos, habilidades, actitudes y valores necesarios para realizar una docencia de calidad (Bozu \& Canto, 2009). La evaluación del desempeño de los docentes es un recurso eficaz para la mejora de los procesos educativos. Riera \& Sansevero (2009) afirman que las competencias pedagógicas del docente deben responder las exigencias de la sociedad actual. En la educación, además de la función docente como mediadora del aprendizaje, también debe generar competencias pedagógicas. Por otra parte Zabalza (2003) considera que el docente de educacion superior debe desarrollar las siguientes competencias: a) planificar el proceso de enseñanza - aprendizaje, b) seleccionar y preparar contenidos disciplinares, c) ofrecer información y explicaciones comprensibles, d) manejo de nuevas tecnologías, e) diseñar la metodología y organizar las actividades, f) comunicarse con los alumnos, g) ofrecer tutoría, h) evaluar, i) investigar, j) Identificarse con la institución, y k) trabajar en equipo. 
Oramas et al. (2013) afirman que en la evaluación de las competencias del docente deben considerarse los conocimientos, las actitudes, los valores de su desempeño, sus principales fuentes de evidencia y los criterios para la medición.

A partir de este análisis se define como desempeño del docente para esta investigación el nivel de desarrollo de las competencias docentes entre la cuales consideraremos las siguientes: conocimientos, actitudes, selección y preparación de contenidos, ofrecer información y explicaciones comprensibles, manejo de nuevas tecnologías y comunicación con los alumnos.

\section{Infraestructura}

Otro aspecto a considerar para evaluar la satisfaccion del estudiante de educación superior es la infraestructura de la Institución, ya que esta permite visualizar las circuntancias físicas bajo las cuales ocurre el aprendizaje. El Laboratorio Lationamericano de Evaluación de la Calidad de la Educación (2016), considera como características de infraestructura las instalaciones físicas y servicios de recursos físicos las cuales están vinculadas significativamente con el aprendizaje.

Derivado de lo anterior se considera la infraestructura como las instalaciones físicas de la universidad donde ocurre el aprendizaje y el servicio de recursos físicos a los que tienen acceso los alumnos de la educación superior.

\section{Servicios de apoyo}

Salinas \& Martínez (2007), señalan que el ambiente que rodea el proceso de enseñanza aprendizaje incide en la calidad del servicio, por lo que es indispensable para que el proceso se logre satisfactoriamente considerar lo siguiente: aulas, biblioteca, cafetería, jardines, procesos administrativos, campos deportivos, estacionamientos, y limpieza en todos estos espacios. Por otro lado, García (2010) afirma que las universidades requieren considerar programas como tutorías, actividades deportivas, actividades de responsabilidad social, artísticas y culturales para lograr una formación integral del alumno. 
En relación a lo anterior, para esta investigación se considera a la variable servicios de la institución como los servicios ofrecidos por la Universidad a los alumnos en lo relativo a biblioteca, centro de cómputo, cafetería, procesos administrativos, servicio médico, actividades deportivas, artísticas, culturales, de responsabilidad social y tutorías, aspectos que serán considerados en la integración de la variable servicios.

\section{Pregunta central de investigación}

¿En qué grado inciden las unidades de aprendizaje, la evaluación del proceso de enseñanza aprendizaje, el desempeño de los profesores, los servicios de apoyo y la infraestructura en la satisfacción de alumnos de educación superior de programas de calidad?

\section{Objetivo general de la investigación}

Determinar cuáles son los factores clave que inciden en el grado de satisfacción en los alumnos que realizan sus estudios de educación superior en programas de calidad del Centro Universitario Sur de la Universidad Autónoma de Tamaulipas.

\section{Hipótesis general de investigación}

Las unidades de aprendizaje, la evaluación del proceso de enseñanza aprendizaje, el desempeño de los profesores, los servicios de la institución y la infraestructura están relacionados positivamente con la satisfacción de los alumnos de educación superior.

\section{Metodología}

La metodología que se aplica para esta investigación es de tipo cuantitativa no experimental, correlacional y de diseño transversal. Este tipo de estudios tiene como finalidad conocer la relación o grado de asociación que exista entre dos 0 más conceptos, categorías o variables en un contexto en particular. (Hernández et al., 2010). El universo de esta investigación son los 
alumnos inscritos en el periodo escolar 2017 - 2018 en programas de calidad del Centro Universitario Sur de la Universidad Autónoma de Tamaulipas, que asciende a 5,654 alumnos, determinándose una muestra representativa de 360 alumnos mediante el método de Muestra Probabilística Estratificada (Levine et al., 2006).

Derivado de lo anterior, se realizó la prueba piloto del instrumento con la recolección de datos que consta de la aplicación de una encuesta de opinión a 90 alumnos, destinada a medir la satisfacción de los alumnos, y la relación de la satisfacción con las variables unidades de enseñanza aprendizaje, la metodología de evaluación, el desempeño de los profesores, la infraestructura y los servicios de la institución. El cuestionario está conformado por 52 ítems en una escala de Likert de siete niveles.

La validación y confiabilidad del instrumento se realizó en tres etapas: la primera etapa se llevó a cabo con un grupo de cinco expertos, quienes se dieron a la tarea de validar el contenido de los ítems y su relación con las variables. Para la segunda etapa, participaron un grupo de cinco expertos quienes determinaron la importancia de los ítems en cada variable. La tercera etapa fue sujeta a un análisis de alfa de cronbach.

\section{Resultados de la prueba piloto}

Para efectos de validar las variables de la investigación, se realizó un análisis factorial por variable considerando inicialmente el contraste de esfericidad de Bartlett y de medida de KMO para determinar la independencia de las variables y si la técnica de análisis factorial es aplicable en este caso, ello con apoyo del software SPSS, lo cual resultó aceptable. 
Tabla 1: Prueba de KMO y Bartlett

\begin{tabular}{|c|c|c|c|c|c|c|c|}
\hline Test & & $\mathrm{X} 1$ & $\mathrm{X} 2$ & X3 & $\mathrm{X} 4$ & $\times 5$ & $Y$ \\
\hline $\begin{array}{l}\text { Medida } \\
\text { Kaiser-Meyer- } \\
\text { Olkin de } \\
\text { adecuación } \\
\text { de muestreo }\end{array}$ & & .843 & .856 & .868 & .859 & .769 & .757 \\
\hline \multirow[t]{3}{*}{$\begin{array}{l}\text { Prueba de } \\
\text { esfericidad de } \\
\text { Bartlett }\end{array}$} & $\begin{array}{c}\text { Aprox. } \\
\text { Chi- } \\
\text { cuadrado }\end{array}$ & 343.474 & 436.708 & 574.723 & 659.594 & 721.336 & 246.632 \\
\hline & $\mathrm{gl}$ & 21 & 21 & 45 & 45 & 78 & 10 \\
\hline & Sig. & .000 & .000 & .000 & .000 & .000 & .000 \\
\hline
\end{tabular}

Nota1: Fuente elaboración propia con datos obtenidos en SPSS.

Posteriormente se realizó un análisis para determinar el coeficiente de variación y así tener las variables que presentan mayor variación con respecto a otras. Al aplicar el método de extracción de componentes principales en el análisis factorial con rotación varimax se obtuvieron los resultados que se muestran en la Tabla 2 para cada una de las variables objeto de estudio.

Tabla 2. Varianza total explicada

\begin{tabular}{ccccc}
\hline Variable & Componente & Total & $\begin{array}{c}\text { Autovalores iniciales } \\
\text { \% de varianza }\end{array}$ & \% acumulado \\
\hline X1 & 1 & 4.228 & 60.394 & 60.394 \\
X2 & 1 & 4.682 & 66.885 & 66.885 \\
X3 & 1 & 5.650 & 56.504 & 56.504 \\
X4 & 1 & 6.124 & 61.243 & 61.243 \\
X5 & 1 & 5.444 & 41.879 & 41.879 \\
Y & 1 & 3.282 & 65.643 & 65.643 \\
\hline
\end{tabular}

Nota2. Fuente: Elaboración propia utilizando el software SPSS

En la Tabla 2 se observan los valores que expresan la cantidad de la varianza total, que está explicada por cada factor. En dicha tabla se 
presentan los valores mayores que 1 y se eliminan los valores menores de 1 en cada variable. De esta manera el procedimiento extrae un factor por cada variable. Con los que respecta a X1 los ítems explican el 60\%, para X2 los ítems explican en $67 \%$, para X3 los ítems explican el $57 \%$, para X4 los ítems explican el $61 \%$, para $X 5$ los ítems explican el $41 \%$ y para $Y$ los ítems explican el $66 \%$.

En la Tabla 3, se observan las cargas de los factores obtenidos, de las cuales se consideran representativas aquellas ponderaciones cuyo valor sea superior a 0.70 , pues las ponderaciones menores indican una escasa representatividad de los ítems en la variable. Esta tabla permitió identificar los ítems que integran cada variable lo cual, junto con el conocimiento de los factores teóricos subyacentes, resultaron pertinentes para plantear el modelo.

Tabla 3. Matriz de componentes.

\begin{tabular}{|c|c|c|c|c|c|}
\hline Variable & İtem & Carga & Variable & Ítem & Carga \\
\hline \multirow[t]{7}{*}{$\mathrm{X} 1$} & $\mathrm{P} 1$ & 0.782 & $\mathrm{X} 2$ & P8 & 0.836 \\
\hline & P2 & 0.841 & & P9 & 0.783 \\
\hline & P3 & 0.842 & & P10 & 0.814 \\
\hline & P4 & 0.797 & & P11 & 0.858 \\
\hline & P5 & 0.755 & & P12 & 0.839 \\
\hline & P6 & 0.778 & & $\mathrm{P} 13$ & 0.83 \\
\hline & & & & P14 & 0.761 \\
\hline \multirow[t]{4}{*}{$\mathrm{X} 3$} & P17 & 0.84 & $X 4$ & P29 & 0.804 \\
\hline & P18 & 0.872 & & P30 & 0.863 \\
\hline & P19 & 0.768 & & P31 & 0.706 \\
\hline & P23 & 0.744 & & P33 & 0.723 \\
\hline \multirow[t]{5}{*}{$x 5$} & P43 & 0.867 & Y & P48 & 0.846 \\
\hline & P44 & 0.886 & & P49 & 0.813 \\
\hline & P45 & 0.864 & & P50 & 0.806 \\
\hline & P46 & 0.811 & & P51 & 0.86 \\
\hline & P47 & 0.818 & & P52 & 0.719 \\
\hline
\end{tabular}

Nota3. Fuente: Elaboración propia utilizando el software SPSS

En la Tabla 4, los valores obtenidos para el Alpha de Cronbach se encuentran en el rango de 0.8 a 0.9 por lo tanto se consideran aceptables, de tal forma que los constructos corresponden a la valoración de las variables. 
Tabla 4. Alpha de Cronbach

\begin{tabular}{lc}
\hline \multicolumn{1}{c}{ Variable } & Resultados \\
\hline Unidades de enseñanza aprendizaje & .887 \\
Metodología de evaluación del proceso enseñanza aprendizaje & .916 \\
Desempeño del docente & .869 \\
Servicios de apoyo & .871 \\
Infraestructura & .922 \\
Satisfacción del alumno & .867 \\
\hline
\end{tabular}

Nota4. Fuente: Elaboración propia utilizando el software SPSS

Para efectos de conocer la vinculación de cada variable de la investigación con respecto a la satisfacción de los alumnos se realizó un análisis de regresión lineal. Con base en los postulados de Rodriguez \& Mora (2001), utilizando para el efecto el paquete estadístico SPSS. El modelo generado arrojó una $\mathrm{R}^{2}$ de 0.624 , la cual resultó aceptable ya que indica que el modelo se representa $62.4 \%$ de la varianza explicada. Esto se muestra en la Tabla 5.

Tabla 5. Resumen del modelo

\begin{tabular}{cccccc}
\hline Modelo & $\mathbf{R}$ & R cuadrado & $\begin{array}{c}\text { R cuadrado } \\
\text { ajustado }\end{array}$ & $\begin{array}{c}\text { Error estándar } \\
\text { de la estimación }\end{array}$ & $\begin{array}{c}\text { Durbin- } \\
\text { Watson }\end{array}$ \\
\hline 1 & .790 & .624 & .601 & .5027344384 & 2.484 \\
\hline
\end{tabular}

Nota 5. Fuente: Elaboración propia utilizando el software SPSS v21

En relación a la prueba Durbin Watson, también se muestra en la Tabla 5. El valor resultante fue de 2.485 , lo que excluye que puede existir auto correlación serial entre los residuos.

La Tabla 6 muestra el ANOVA del modelo de regresión, donde se puede observar el estadístico $F$ con un valor de 27.829 y un nivel de significación de .000, lo cual es estadísticamente aceptable. 
Tabla 6. ANOVA

\begin{tabular}{lccccc}
\hline Modelo & $\begin{array}{c}\text { Suma de } \\
\text { cuadrados }\end{array}$ & $\mathbf{g l}$ & Media cuadrática & $\mathbf{F}$ & Sig. \\
\hline 1 Regresión & 35.168 & 5 & 7.034 & 27.829 & $.000^{\circ}$ \\
Residuo & 21.230 & 84 & .253 & & \\
Total & 56.398 & 89 & & & \\
\hline
\end{tabular}

Nota 6. a. Variable dependiente: $Y$

b. Predictores: (Constante), INFRA, UEA, SERVICIOS, DESEMP, METOD

Fuente: Elaboración propia utilizando el software SPSS

En la Tabla 7, se presenta los resultados de la multicolinealidad, donde se observa que algunas variables predictoras incluidas en el modelo están correlacionadas con otras. Así se procedió a eliminar las variables de desempeño docente y metodología de evaluación.

Tabla 7. Multicolinealidad

\begin{tabular}{ccccccc}
\hline Variable & $\mathbf{Y}$ & UEA & METOD & DESEM & INFRA & SERVICIOS \\
\hline Y & 1.000000 & 0.533031 & 0.528811 & 0.521278 & 0.627562 & 0.678508 \\
UEA & 0.533031 & 1.000000 & 0.768770 & 0.670934 & 0.457449 & 0.402732 \\
METOD & 0.528811 & 0.768770 & 1.000000 & 0.704205 & 0.565325 & 0.494951 \\
DESEM & 0.521278 & 0.670934 & 0.704205 & 1.000000 & 0.576902 & 0.567673 \\
INFRA & 0.627562 & 0.457449 & 0.565325 & 0.576902 & 1.000000 & 0.604359 \\
SERVICIOS & 0.678508 & 0.402732 & 0.494951 & 0.567673 & 0.604359 & 1.000000 \\
\hline
\end{tabular}

Nota 7 Fuente: Elaboración propia utilizando el software SPSS

En la Tabla 8, se presenta los resultados del test White utilizado para la prueba de heterocedasticidad, donde se observa que en esta prueba piloto hay evidencia de heterocedasticidad, por lo tanto, se procede a realizar la corrección por errores estándar robustos de White, obteniendo los resultados como se muestran en la Tabla 9.

Tabla 8. Heterocedasticidad Test

\begin{tabular}{llll}
\hline Estadístico & Valor & Tipo de Probabilidad & Valor \\
\hline F-statistic & 2.534725 & Prob. F(9,80) & 0.0130 \\
Obs*R-squared & 19.96962 & Prob. Chi-Square(9) & 0.0181 \\
Scaled explained SS & 40.13732 & Prob. Chi-Square(9) & 0.0000 \\
\hline
\end{tabular}

Nota 8. Fuente: Elaboración propia utilizando el software Eviews 
Tabla 9. Errores estándar robustos de White

\begin{tabular}{|c|c|c|c|c|}
\hline Variable & Coeficiente & Error Std. & Estatistico-t & Prob. \\
\hline$C$ & 0.219886 & 0.214078 & 1.027131 & 0.3072 \\
\hline UEA & 0.274613 & 0.086790 & 3.164109 & 0.0022 \\
\hline INFRA & 0.286942 & 0.071405 & 4.018497 & 0.0001 \\
\hline SERVICIO & 0.349182 & 0.074145 & 4.709449 & 0.0000 \\
\hline R-squared & 0.620094 & & Mean dependent var & 2.964096 \\
\hline Adjusted R-squared & 0.606842 & & S.D. dependent var & 0.690718 \\
\hline S.E. of regression & 0.433096 & & Akaike info criterion & 1.207713 \\
\hline Sum squared resid & 16.13123 & & Schwarz criterion & 1.318816 \\
\hline Log likelihood & -50.34710 & & Hannan-Quinn criter. & 1.252516 \\
\hline F-statistic & 46.79066 & & Durbin-Watson stat & 2.130840 \\
\hline $\operatorname{Prob}$ (F-statistic) & 0.000000 & & & \\
\hline
\end{tabular}

Nota 9. Fuente: Elaboración propia utilizando el software Eviews

De acuerdo a lo anterior se establece la siguiente ecuación:

$$
Y=0.219886+0.274613(x 1)+0.349182(x 2)+0.286942(x 3)
$$

donde:

$Y=$ Satisfacción de los alumnos

$x 1=$ Unidades de enseñanza aprendizaje

$x 2=$ Servicios de apoyo

$x 3=$ Infraestructura

Esta ecuación indica que, de las cinco variables en cuestión, tres de ellas resultaron significativas con el siguiente orden de importancia: servicios de apoyo, infraestructura, y unidades de enseñanza aprendizaje. La metodología de evaluación del proceso de enseñanza aprendizaje y el desempeño del docente no fueron estadísticamente significativas; se espera ampliar las observaciones para analizar nuevamente el modelo.

\section{Conclusiones}

Esta investigación que se considera de carácter preliminar con base en una prueba piloto es la base para el análisis de una muestra representativa de la 
satisfacción de los estudiantes del Centro Universitario Sur de la Universidad Autónoma de Tamaulipas, que se llevará a cabo posteriormente. Se desarrolló con base en un enfoque cuantitativo, fundamentado en el análisis estadístico de datos recolectados para la prueba piloto y tomando en consideración la revisión de literatura al respecto.

De acuerdo con Arancibia et al. (2013) para evaluar la calidad del servicio es necesario considerar la percepción del cliente, por lo que en este estudio propone un instrumento para la medición de la satisfacción de los alumnos de educación superior, con la finalidad de conocer cuáles son variables clave que impactan en la calidad educativa desde la perspectiva de los alumnos.

El análisis de los resultados de la prueba piloto indica que de las cinco variables propuestas tres de ellas fueron significativas, y apoyan la satisfacción de los alumnos, con el siguiente orden de importancia:

1) servicios de apoyo (los servicio que brinda el personal de la Universidad a los alumnos en la atención de los tramites escolares, becas, tutorías por mencionar algunos),

2) Infraestructura (la disponibilidad de espacios y áreas para el desarrollo integral del alumnos y funcionamiento adecuado de las instalaciones de la Universidad),

3) Unidades de enseñanza aprendizaje (los contenidos de las materias, así como el cumplimiento de estos, y la duración horas por semana de la materia) las cuales inciden positivamente en la satisfacción de los alumnos.

Resultados con un mayor alcance se podrán conocer una vez que se lleve a cabo la investigación definitiva sobre este caso de estudio, con base en la muestra representativa de los alumnos de licenciatura inscritos en los programas de calidad del Centro Universitario de la Zona Sur de la Universidad Autónoma de Tamaulipas. 


\section{Anexo:}

\section{ENCUESTA SOBRE EL SERVICIO DE EDUCACIÓN SUPERIOR}

La presente encuesta forma parte de una investigación doctoral, y tiene como propósito conocer el grado de satisfacción de los alumnos de nivel superior con los servicios que le ofrece la Universidad Autónoma de Tamaulipas en el Centro Universitario Sur. Los resultados se presentaran por dependencia y globales al concluir el estudio. Los datos recolectados serán tratados con estricta confidencialidad, mucho le agradecería apoyarnos con sus respuestas.

FACULTAD:

CARRERA:

\section{DATOS DEL PARTICIPANTE}

SEXO: SEMESTRE

INSTRUCCIONES: A continuación se presentan una serie de preguntas relacionadas con el servicio que te ofrece la universidad. Por favor marca con una $\mathrm{X}$ la respuesta que refleje tu nivel de satisfacción o insatisfacción, de acuerdo a la siguiente escala:
(1) Extremadamente insatisfecho
(2) Muy insatisfecho
(3) Insatisfecho
(4) Neutro
(5) Satisfecho
(6) Muy satisfecho
(7) Extremadamente satisfecho

UNIDADES DE APRENDIZAJE

\begin{tabular}{|c|c|c|c|c|c|c|c|c|}
\hline \multicolumn{2}{|r|}{ ¿Qué tan satisfecho te encuentras con los siguientes aspectos: } & 1 & 2 & 3 & 4 & 5 & 6 & 7 \\
\hline 1 & $\begin{array}{l}\text { La explicación de los objetivos de las materias que has cursado en los últimos dos } \\
\text { años. }\end{array}$ & & & & & & & \\
\hline 2 & El contenido de las materias que has cursado en los últimos dos años. & & & & & & & \\
\hline 3 & $\begin{array}{l}\text { El cumplimiento de los contenidos de las materias que has cursado en los últimos } \\
\text { dos años. }\end{array}$ & & & & & & & \\
\hline 4 & $\begin{array}{l}\text { El cumplimiento de los objetivos establecidos en el programa de las materias que } \\
\text { has cursado en los últimos dos años. }\end{array}$ & & & & & & & \\
\hline 5 & $\begin{array}{l}\text { La duración de los programas de las materias que has cursado en los últimos dos } \\
\text { años. (horas por semana) }\end{array}$ & & & & & & & \\
\hline 6 & $\begin{array}{l}\text { La presentación estructurada de las materias que has cursado en los últimos dos } \\
\text { años. }\end{array}$ & & & & & & & \\
\hline 7 & $\begin{array}{l}\text { La bibliografía recomendada en el programa de las materias que has cursado en } \\
\text { los dos últimos años. }\end{array}$ & & & & & & & \\
\hline
\end{tabular}

METODOLOGÍA DE EVALUACIÓN DEL PROCESO DE ENSEÑANAZA APRENDIZAJE

\begin{tabular}{|c|c|c|c|c|c|c|c|c|}
\hline \multicolumn{2}{|r|}{ ¿Qué tan satisfecho te encuentras con los siguientes aspectos: } & 1 & 2 & 3 & 4 & 5 & 6 & 7 \\
\hline 8 & El sistema de evaluación utilizado. & & & & & & & \\
\hline 9 & $\begin{array}{l}\text { Los criterios de evaluación (rúbricas o listas de cotejo) que se utilizan para la } \\
\text { evaluación. }\end{array}$ & & & & & & & \\
\hline 10 & El material didáctico utilizado en las clases. & & & & & & & \\
\hline 11 & Las tareas y trabajos asignados. & & & & & & & \\
\hline 12 & Los exámenes que te aplican. & & & & & & & \\
\hline 13 & La retroalimentación que te brindan respecto a tu progreso en la clase. & & & & & & & \\
\hline 14 & La atención individualizada que te brindan en clase, cuando la requieres. & & & & & & & \\
\hline
\end{tabular}


DESEMPEÑO DE LOS PROFESORES

\begin{tabular}{|c|c|c|c|c|c|c|c|c|}
\hline & ¿Qué tan satisfecho te encuentras con los siguientes aspectos: & 1 & 2 & 3 & 4 & 5 & 6 & 7 \\
\hline 15 & El conocimiento y dominio de los temas abordados. & & & & & & & \\
\hline 16 & La capacidad de respuesta a las dudas que planteas. & & & & & & & \\
\hline 17 & La asistencia a clases del profesor. & & & & & & & \\
\hline 18 & La puntualidad a clases del profesor. & & & & & & & \\
\hline 19 & La apariencia del profesor durante la clase. & & & & & & & \\
\hline 20 & El trato que el profesor da a los alumnos durante la clase. & & & & & & & \\
\hline 21 & La comunicación del profesor con el grupo. & & & & & & & \\
\hline 22 & La educación y experiencia académica del profesor. & & & & & & & \\
\hline 23 & El manejo de grupo que tiene el profesor. & & & & & & & \\
\hline 24 & La utilización del profesor de nuevas tecnologías en la clase. & & & & & & & \\
\hline
\end{tabular}

\section{SERVICIO DE APOYO}

\begin{tabular}{|c|c|c|c|c|c|c|c|c|}
\hline \multicolumn{2}{|r|}{ ¿Qué tan satisfecho te encuentras con los siguientes aspectos: } & 1 & 2 & 3 & 4 & 5 & 6 & 7 \\
\hline 25 & El servicio que recibes del personal en centro de cómputo. & & & & & & & \\
\hline 26 & El servicio que recibes del personal biblioteca y laboratorios. & & & & & & & \\
\hline 27 & El servicio que recibes del personal laboratorios. & & & & & & & \\
\hline 28 & El servicio que recibes con tus trámites escolares. & & & & & & & \\
\hline 29 & La atención individualizada que te brindan en la tutoría. & & & & & & & \\
\hline 30 & La atención individualizada que te brindan en la asesoría académica. & & & & & & & \\
\hline 31 & El servicio de trámite de becas, que te brindan. & & & & & & & \\
\hline 32 & El servicio de trámite de movilidad académica, que te brindan. & & & & & & & \\
\hline 33 & El servicio de bolsa de trabajo, que te brindan. & & & & & & & \\
\hline 34 & La atención a las quejas. & & & & & & & \\
\hline
\end{tabular}

\section{INFRAESTRUCTURA}

\begin{tabular}{|c|c|c|c|c|c|c|c|c|}
\hline & an satisfecho te encuentras con los siguientes aspectos: & 1 & 2 & 3 & 4 & 5 & 6 & 7 \\
\hline 35 & Las instalaciones (aulas, espacios de estudio) de la universidad. & & & & & & & \\
\hline 36 & Las instalaciones deportivas de la universidad. & & & & & & & \\
\hline 37 & $\begin{array}{l}\text { El funcionamiento del aire acondicionado en el aula. } \\
\text { (funciona adecuadamente y sin problemas la mayor parte del tiempo) }\end{array}$ & & & & & & & \\
\hline 38 & $\begin{array}{l}\text { La iluminación del aula. } \\
\text { (es suficiente y adecuada para el desarrollo de la clase) }\end{array}$ & & & & & & & \\
\hline 39 & $\begin{array}{l}\text { El equipo de apoyo audiovisual e informático. } \\
\text { (se encuentra en óptimas condiciones) }\end{array}$ & & & & & & & \\
\hline 40 & La condición de los baños. & & & & & & & \\
\hline 41 & La disponibilidad de acervo bibliográfico para consulta en la biblioteca. & & & & & & & \\
\hline 42 & La conexión a internet. & & & & & & & \\
\hline 43 & El funcionamiento del equipo de los laboratorios de la universidad. & & & & & & & \\
\hline 44 & La actualización del equipo de los laboratorios de la universidad. & & & & & & & \\
\hline 45 & La disponibilidad de material en los laboratorios de la universidad. & & & & & & & \\
\hline 46 & El funcionamiento del equipo de cómputo. & & & & & & & \\
\hline 47 & La actualización del equipo de cómputo. & & & & & & & \\
\hline
\end{tabular}

\begin{tabular}{|c|c|c|c|c|c|c|c|c|}
\hline \multicolumn{2}{|c|}{ En general } & 1 & 2 & 3 & 4 & 5 & 6 & 7 \\
\hline 48 & ¿Cómo percibes el servicio que te brinda la universidad? & & & & & & & \\
\hline 49 & $\begin{array}{l}\text { ¿Qué percepción tienes de la pertinencia de lo estudiado para incorporarte al } \\
\text { mercado laboral? }\end{array}$ & & & & & & & \\
\hline 50 & $\begin{array}{l}\text { ¿Qué percepción tienes de tienes de la demanda de los egresados de tu carrera, } \\
\text { en el mercado laboral? }\end{array}$ & & & & & & & \\
\hline 51 & $\begin{array}{l}\text { ¿Qué tan satisfecho estas con la atención del personal de la universidad que te } \\
\text { brinda algún servicio? }\end{array}$ & & & & & & & \\
\hline \multicolumn{2}{|c|}{ Del 1 al 7 califica los siguientes aspectos: } & & & & & & & \\
\hline 52 & Prestigio de la universidad en tu comunidad & & & & & & & \\
\hline
\end{tabular}

Gracias por tu participación. 


\section{Referencias}

Alvarado, E., Morales, D. \& Aguayo, E. (2016). Percepción de la calidad educativa: caso aplicado a estudiantes de la Universidad Autónoma de Nuevo León y del Instituto Tecnológico de Estudios Superiores de Monterrey, Revista de la Educación Superior, 45(180), 55-74.

Alvarez, J., Chaparro, E. \& Reyes, D. E. (2015). Estudio de la satisfacción de los estudiantes con los servicios educativos brindados por Instituciones de Educación Superior del Valle de Toluca, Revista Iberoamericana sobre Calidad, Eficacia y Cambio en Educación, 13 (2),5-26.

Arancibia-Carvajal, S., Leguina, A. \& Espinosa Zamorano, P. (2013). Factores determinantes en la percepción de la imagen y calidad de servicio y sus efectos en la satisfaccion del cliente. Un caso aplicado a la banca chilena, Revista de Ciencias Sociales, 19 (2), 255-267.

Bozu, Z. \& Canto Herrera, P. (2009). El profesorado universitario en la sociedad del conocimiento: competencias profesionales docentes, Revista de Formación e Immovación Educativa Universitaria, 2(2), 87-97.

Brunner, J. J. \& Elacqua, G. . (2004). Factores que inciden en una educación efectiva. Evidencia internacional, La Educación: Revista Interamericana de Desarrollo Educativo, 48-49 (139-140), 1-11.

Darlaston-Jones, D., Pike, L., Cohen, L., Young, A., Haunold, S. \& Drew, N. (2003). Are they being served? Students' expectations of higher education, Issues in Educational Research, 13(1), 31-52.

Díaz, Á. (2014). Construcción de programas de estudio en la perpectiva del enfoque de desarrollo de competencias, Perfiles Educativos, 36(143), 142-162.

Faganel, A. \& Dolinsek, S. (2012). Quality management system in higher education. En Vodovnik, Z. (Ed.) Intellectual capital and knowledge management. Portoroz: Faculty of Management Koper, University of Primorska, 245-251.

García, S. L. (2010). El papel de la tutoría en la formación integral del universitario, Tiempo de educar, 11(21), 31-56.

Gento, S. \& Vivas, M. (2003). El SEUE: un instrumento para conocer la satisfacción de los estudiantes universitarios con su educación, Acción pedagógica, 12(2), 16-27.

Gobierno del Estado de Tamaulipas. (2017). Plan estatal de desarrollo 2016-2022. Victoria, Tamaulipas, México.

González, M., Pino, M. \& Penado, M. (2017). Estudio de la satisfacción percibida por los estudiantes de la UNED cons su vida universitaria, Revista Iberoamericana de Educación a Distancia, 20(1), 243-260.

Hamodi, C., López, V. \& López, A. (2015). Medios, técnicas e instrumentos de evaluación formativa y compartida del aprendizaje en educación superior, Perfiles educativos, 37(147), 144-161.

Hernández, R., Fernández, C. \& Baptista, P. (2010). Metodología de la investigación. México: Mc Grall Hill. 
Hill, F. M. (1995). Managing service quality in higher education: the role of the consumer as primary consumer, Quality Assurance in Higher Education, 3(3), 10- 21.

Jiménez, A., Terriquez, B., \& Robles, F. J. (2011). Evaluación de la satisfacción académica de los estudiantes de la Universidad Autónoma de Nayarit, Revista Fuente, 3(6), 46-56.

Keogh, K. M. \& Stevenson, K. (2001, abril). Student expectations of tutor support: An expectations led quality assurance model, Revista Openlearning, 11(1),22-30

Laboratorio Lationamericano de Evalucación de la Calidad de la Educación. (2016). Tercer Estudio Regional Comparativo y Explicativo. Santiago: OREALC/UNESCO.

Levine, D. M., Krehbiel, T. C. \& Berenson, M. L. (2006). Estadística para administración. Cd. México: PERSON Educación.

Mejías, A. \& Martínez, D. (2009). Desarollo de un instrumento para medir la satisfacción estudiantil en educación superior, Docencia Universitaria, 10(2), 29-47.

Narasimhan, K. (1997). Improving teaching and learning: Perceptions minus expectations gap analysis approach, Training for Quality, 5(3), 121-125.

Oramas, R., Jordán, T. \& Valcárcel, N. (2013). Competencias y desempeño profesional pedagógico hacia un modelo del profesor de la carrrera de Medicina, Revista Cubana de Educación Media Superior, 27(1), 123-134.

Parasuraman, A., Zeithaml, V. \& Berry, L. (1985). A conceptual model of service quality and its implications for future research, Journal of Marketing, 49(4), 41-50.

Pichardo, M., García, A., De la Fuente, J. \& Justicia, F. (2007). El estudio de las expectativas en la universidad: análisis de trabajos empíricos y futuras líneas de investigación, Revista electrónica de investigación educativa, 9(1), 1-16.

Riera, L. \& Sansevero, I. (2009). Competencias pedagógicas del docente democrático en la educación básica, Revista de Estudio Interdisciplinarios en Ciencias Sociales, 11(2), 211-228.

Rodríguez, M. J. \& Mora, R. (2001). Estadística Informática: casos y ejemplos con el SPSS. San Vicente del Raspeig: Universidad de Alicante. Servicio de Publicaciones.

Salinas, A. \& Martínez, P. (2007). Principales factores de satisfacción entre los estudiantes universitarios, Revista Internacional de Ciencias Sociales y Humanidades, 17(1), 163-192.

Sander, P., Stevenson, K., King, M. \& Coates, D. (2000). University Students' Expectations of Teaching, Studies in Higher Education, 25(3), 309-323.

Santos, M. A (1993). La evaluación: un proceso de diálogo, comprensión y mejora. Málaga: Aljibe, Revista investigación en la escuela, (20), 23-35.

Weinstein, J. (2002). Calidad y Gestión: Condiciones y Desafíos, Revista Pensamiento Educativo, 31(2), 50-71.

Zabalza, M. A. (1987). Diseño y desarrollo curricular. Madrid: Narcea Ediciones.

Zabalza, M. A. (2003). Competencias docentes del profesorado universitario. Calidad y desarrollo profesional. Madrid: Narcea Ediciones. 